\title{
Long-term Follow-up in Adults with Coeliac Disease: Predictors and Effect on Health \\ Outcomes
}

Henna Pekki ${ }^{1,2}$, Katri Kaukinen ${ }^{1-3}$, Tuire Ilus ${ }^{1,4}$, Markku Mäki $^{5}$, Heini Huhtala ${ }^{6}$, Kaija Laurila ${ }^{5}$, Kalle Kurppa ${ }^{2,5}$

${ }^{1}$ Celiac Disease Research Centre, University of Tampere and Tampere University Hospital, Tampere, Finland

${ }^{2}$ Faculty of Medicine and Life Sciences, University of Tampere, Tampere, Finland ${ }^{3}$ Department of Internal Medicine, Tampere University Hospital, Tampere, Finland

${ }^{4}$ Department of Gastroenterology and Alimentary Tract Surgery, Tampere University Hospital, Tampere, Finland

${ }^{5}$ Tampere Center for Child Health Research, University of Tampere and Tampere University Hospital, Tampere, Finland

${ }^{6}$ Faculty of Social Sciences, University of Tampere, Tampere, Finland

Correspondence to Kalle Kurppa, MD, $\mathrm{PhD}$

Faculty of Medicine and Life Sciences, University of Tampere, FIN-33014, Tampere, Finland. E-mail: kalle.kurppa@uta.fi

The authors report no conflict of interest

Word count: 2981

Abbreviations: EmA, Endomysium antibodies, GSRS, Gastrointestinal Symptom Rating Scale; PGWB, Psychological Well-Being Index; SF-36, Short-Form 36; TG2ab, transglutaminase 2 antibodies 
Introduction: Current guidelines recommend regular follow-up in coeliac disease, but effect of this on long-term health outcomes remains unclear.

Aims: To evaluate predictors and significance of long-term follow-up

Methods: Altogether 677 previously diagnosed coeliac disease patients were recruited for a nationwide health survey. Medical data were gathered through interviews and patient records. Current symptoms and quality of life were assessed by validated questionnaires and blood samples were drawn for serology. All variables were compared between patients with and without long-term (>2 years) follow-up.

Results: Only 15\% had long-term follow-up, median duration 10 years. Predictors $(\mathrm{p}<0.05)$ for the follow-up were immunological (35\% vs. 24\%) and circulatory (20\% vs. 12\%) comorbidities, whereas it was less common in subjects with musculoskeletal (23\% vs. $34 \%$ ) comorbidity and those not belonging to any at-risk group (16\% vs. $27 \%)$. Patients with or without follow-up had comparable age, adherence and ability to manage a gluten-free diet and frequency of seropositivity. Also questionnaire scores paralleled, but those without follow-up reported more overall symptoms (16\% vs. 26\%). Most patients in both groups wished for follow-up.

Conclusion: Only a minority of patients had regular follow-up. However, patients with and without the follow-up were comparable in most long-term outcomes, indicating that it might not be always necessary. The results call for more personalized follow-up policies in coeliac disease.

Key words: Gluten-free diet; Treatment, Quality of life; Symptoms; Complications 


\section{Introduction}

Coeliac disease is a lifelong gluten-induced autoimmune enteropathy with a prevalence up to $2 \%$ in Caucasian populations (1). The only treatment is a strict gluten-free diet, the initiation of which usually elicits a clear clinical and histological response and disappearance of the diseasespecific autoantibodies (2). Unfortunately, the high cost and restrictive nature of the gluten-free diet predisposes to poor adherence and subsequently to persistent enteropathy and an increased risk of serious complications such as osteoporotic fractures and malignancy (3-6). Moreover, there is a subgroup of patients with a condition called refractory coeliac disease, who do not attain adequate clinical and histological recovery despite a strict diet and thus have particularly poor prognosis (7).

In order to ensure proper adherence and response to the gluten-free diet and to detect possible complications, most current guidelines recommend regular long-term follow-up in coeliac disease even as often as annually $(2,8)$. However, there is a paucity of evidence as to how the follow-up is actually implemented in clinical practice, and whether the absence of follow-up really affects the long-term coping and health of the patients (9-15). Interestingly, in a 15-year follow-up we recently found that the lack of a repeat biopsy one year after coeliac disease diagnosis is not associated with an increased risk of adverse outcomes such as reduced well-being or malignancies (16). This would indicate that the association between the presence of follow-up and the prognosis of coeliac disease is more complex than one might expect.

To further elucidate the significance of regular follow-up to the treatment success in coeliac disease, we conducted a nationwide survey and compared various patient-related and other relevant factors between large cohorts of coeliac disease patients with or without longterm follow-up after diagnosis.

Materials and Methods 


\section{Patients and study design}

A nationwide cross-sectional health survey was conducted in Tampere University Hospital and the University of Tampere. The survey was created to investigate variable aspects of coeliac disease, not just factors associated with the long-term follow-up. The participants were recruited through newspaper advertisements and with the aid of coeliac disease societies. Inclusion criteria were age $\geq 18$ years and a biopsy-proven diagnosis at least two years before enrolment. All participants filled validated questionnaires on current symptoms and quality of life and were interviewed systematically by a physician or a study nurse with expertise in coeliac disease. Patients unable to attain the study center were interviewed by phone and questionnaires were sent by mail. Patient records were reviewed in order to confirm the diagnosis and to complement medical data. In addition, blood samples were drawn for serological measurements, in case of phone interviews this was done at the nearest laboratory. Subjects with dermatitis herpetiformis were excluded owing to their different diagnostic and follow-up protocol, as well as patients with an unclear diagnosis or substantially incomplete medical information. Altogether 677 participants, representing approximately $2 \%$ of the whole Finnish coeliac population (4), were enrolled for further analyses.

After data collection the results were analyzed between subgroups of participants who either had or had not been systematically and regularly assessed for at least two years after the diagnosis by health care for dietary compliance and treatment success. This sorting was based on the patients' own reporting and medical record data. Also possible coeliac disease controls carried out during health care visits for other conditions were included. Subgroup analyses were carried out in patients with only a short-term follow-up $(<2$ years $)$ and those without any follow-up. The findings were compared with our national recommendations. In Finland, the follow-up of coeliac disease is decentralized to primary care, with the general practitioners in charge. If needed, the patient can be referred to a specialist in a reasonable time 
frame. To unify diagnostics and treatment, there are national Current Care Guidelines, which recommend a repeat biopsy one year after diagnosis and regular clinical and serological followup at 2-3-year intervals (Collin 2010).

Patient enrolment and collection of study data were conducted with the permission and according to the guidelines of the Ethical Committee of the Pirkanmaa Hospital District. All participants gave written informed consent.

\section{Clinical characteristics}

The clinical information collected included demographic data, the type and the severity of clinical presentation before diagnosis, duration of symptoms and their current self-experienced persistence. Family history of coeliac disease, presence of coeliac disease-associated and other significant chronic comorbidities were also inquired. The latter included particularly immunological (e.g. asthma, allergies), circulatory (e.g. hypertension, coronary artery disease) and musculoskeletal (e.g. arthritis, fibromyalgia) conditions. Current or previous smoking and whether the coeliac disease diagnosis was made in primary, secondary or tertiary public care or in private care were also inquired. The self-perceived severity of clinical presentation was asked to estimate the burden of symptoms. These were further classified into 1) no symptoms; 2) mild symptoms such as occasionally disturbing gastrointestinal or extra-intestinal symptoms or a combination of them and 3) severe symptoms seriously disturbing daily life, such as recurrent awakenings because of pain or symptoms requiring acute inpatient care (17).

\section{Small-bowel mucosal biopsies}

Data on small-bowel mucosal biopsies were collected from the patient records. Our national diagnostic guidelines for coeliac disease recommend at least four duodenal biopsies to be taken routinely from each patient upon coeliac disease suspicion and during the possible repeat 
endoscopy after one year on a gluten-free diet. The histological specimens are forwarded to the hospitals' pathology department, where the severity of mucosal damage is evaluated in representative and correctly orientated biopsy cuttings. Demonstration of duodenal villous atrophy is required for celiac disease diagnosis. The degree of mucosal lesion has for decades been graded as partial, subtotal or total villous atrophy, these corresponding approximately grades 3a, $3 b$ and $3 c$ in Marsh-Oberhuber classification.

\section{Celiac disease serology}

Values for serum endomysial antibodies (EmA) at diagnosis were gathered from patient records. Furthermore, EmA and transglutaminase 2 antibodies (TG2ab) were measured in all patients at the time of the current study. Indirect immunofluorescence was used for EmA measurements as previously described (18). Titers $1: \geq 5$ were considered positive and diluted until negative to $1: 50,1: 100,1: 200,1: 500,1: 1000,1: 2000$ and 1:4000. These were further classified as low (1:5-1:200) and high positive (1:500-1:4000). TG2ab were tested by commercial ELISA (QUANTA Lite h-tTG IgA, INOVA Diagnostics, San Diego, CA), considering a cut-off $>30.0 \mathrm{U} / 1$ positive. Corresponding IgG-class antibodies were measured in cases of selective IgA deficiency.

\section{Adherence to the gluten-free diet}

Provision of dietary advice at coeliac disease diagnosis was verified by patient interview and from patient records. Current long-term dietary adherence was also inquired by an experienced study nurse/physician with an expertise in coeliac disease and classified as "strict" (minor inadvertent lapses less than a few times a year), "occasional lapses" (lapses less often than once per month) and "normal diet". Long-term adherence was further estimated on the basis of 
coeliac antibody positivity at the time of the present study. Alongside adherence, patient's overall competency in managing the diet and the possible use of purified oats was asked.

\section{Questionnaires}

Short Form 36 Health Survey (SF-36) and Psychological General Well-Being questionnaires (PGWB) were used to assess patients' current self-perceived quality of life and gastrointestinal symptoms at time of study.

SF-36 comprises 36 separate questions, divided into eight sub-dimensions as follows; physical functioning, role limitations due to physical problems, bodily pain, general health, vitality, social functioning, role limitations due to emotional problems and mental health (19-23). Items are re-scored from 0 to 100 , higher scores indicating better health and quality of life.

PGWB is a well-validated and widely used questionnaire both in coeliac disease and in general $(18,20,22,23)$. The 22 separate items can be further divided into six subdimensions measuring anxiety, depression, well-being, self-control, general health and vitality. All items use a 6-grade Likert scale with higher scores representing better well-being and quality of life.

Gastrointestinal symptoms were evaluated by the Gastrointestinal Symptom Rating Scale (GSRS) questionnaire $(18,24)$. It includes 15 separate items which can be added together as a total score and further divided into 5 sub-dimensions measuring abdominal pain, gastroesophageal reflux, indigestion, diarrhea and constipation. The scoring is based on a 7-grade Likert scale, higher scores reflecting more severe gastrointestinal symptom.

Statistical analysis 
Continuous variables are presented as medians with quartiles or ranges. Categorical variables are presented as number of subjects and percentages. Statistical significance in differences between patients with and without long-term follow-up was studied using Mann-Whitney test. Binominal and classified variables were analyzed using Chi-square test. A p-value $<0.05$ was considered significant in all analyses. All analyses were carried out using SPSS 20.0 (IBM Corp. Armonk, NY).

Results

The median age of the whole study cohort was 44 (range 22-89) years and $80 \%$ were women. The median follow-up time for the whole study group was 10 (range 2-38) years. Out of the 677 participants, $99(15 \%)$ had and $578(85 \%)$ had not received long-term follow-up for coeliac disease.

\section{Factors predicting long-term follow-up}

Existence of follow-up was predicted by the presence of immunological ( $35 \%$ vs $24 \%, p=0.020)$ and circulatory ( $20 \%$ vs. $12 \%, \mathrm{p}=0.010)$ comorbidities, whereas it was less common in subjects with coexisting musculoskeletal disease ( $23 \%$ vs. $34 \%, \mathrm{p}=0.045)$. Those not belonging to any at-risk group, such as patients with type I diabetes and those with family history for coeliac disease, were more likely to be without follow-up (Table 1). In contrast, follow-up was not predicted by other co-morbidities (data not shown), gender, site of diagnosis, seropositivity for celiac autoantibodies or severity of histological lesion at diagnosis, symptoms in childhood, clinical presentation or duration of symptoms before diagnosis, family risk of coeliac disease or associated condition and smoking (Table 1). Also age at diagnosis (median 42 vs. 44 years, range $18-75$ vs. $18-71$ years, $\mathrm{p}=0.117$ ) or at present (median 54 vs. 54 years, range $22-81$ vs 
21-89 years, $\mathrm{p}=0.919$ ) did not significantly differ between patients with and without followup.

When looking into the short-term clinical characteristic one year after diagnosis of those patients the data was available, there was no significant association between the presence coeliac autoantibody positivity ( $6.3 \%$ vs. $15.1 \%$, respectively $p=0.343 ; n=95)$ between groups. Neither did the groups differ in the prevalence of histological recovery (fully normalized 53.0\% vs. $60.2 \%, p=0.378 ; n=398$ ) in the follow-up and no-follow up groups after one year on a glutenfree diet and existence of long-term follow.

\section{Long-term outcomes in patients with and without follow-up}

Participants in both follow-up and no-follow up groups had similar dietary adherence and ability to manage the gluten-free diet, as well as use of purified oats (Table 2). There was also no difference between the groups in current positivity for coeliac autoantibodies (Table 2) or in any of the questionnaire scores measuring health-related quality of life (SF-36, PGWB) and gastrointestinal symptoms (GSRS) (Table 3). However, patients without regular long-term follow-up suffered more from current self-reported overall symptoms (Table 2). Despite this difference in significance, there was a correlation between self-reported symptoms and those evaluated by GSRS. Nevertheless, patients with more indigestion, reflux, constipation and abdominal pain in GSRS are more likely to report more severe self-perceived symptoms. In contrast, the amounts of diarrhea and constipation were not associated with the severity of selfperceived symptoms (Data not shown)

Altogether $98 \%$ of patients with regular long-term follow-up wished for it also in the future, and this was also seen in over $80 \%$ of the patients of patients not currently under follow-up (Table 2). Most of these patients wished the follow-up to be organized in public 
healthcare (Figure 1), whereas there was no major difference regarding who should be in charge of the follow-up (Figure 2).

\section{Sub-analysis of patients without any follow-up and short-term follow-up}

Altogether $84(12 \%)$ out of the 677 participants had not received any follow-up for coeliac disease after the diagnosis, while $465(69 \%)$ had been subject only to short-term follow-up ( $<2$ year). Compared to patients receiving long-term follow-up, patients without any follow-up had more current mild symptoms $(27 \%$ vs. $13 \% \mathrm{p}=0.038)$. Furthermore, those without any followup had fewer circulatory diseases $(18 \%$ vs. $35 \%$, respectively, $\mathrm{p}=0.009)$ and a lower desire for regular follow-up ( $85 \%$ vs. $92 \%, p=0.024)$ than those with long-term follow-up.

\section{Discussion}

We found the prevalence of regular follow-up to be only $15 \%$ in Finnish coeliac disease patients (16). This is in stark contrast to our national guidelines that recommend regular long-term clinical and serological follow-up in all patients (Collin 2010). It is also a markedly lower percentage than reported previously. In the early 1990s Bardella et al. (25) reported a followup rate of $77 \%$. Bebb et al. found the corresponding figure to be $62 \%$, although only $6 \%$ of the patients later transferred to primary care received follow-up (26). In a study by Herman et al. $41 \%$ of patients received a follow-up during the first year after, this percentage increasing to $89 \%$ after five years (11). The results by Bardella et al. could be affected by the fact that the patients were investigated during their transition from pediatric to adult care, at which time at least one visit is often scheduled. Another explanation for the different percentages could be related to our practice to refer coeliac disease patients in primary care soon after the diagnosis, whereas most of the previous studies were carried out in tertiary centers. We must, however, 
emphasize that also the majority of our patients received short-term follow-up, including a follow-up biopsy one year after the diagnosis (16).

There are also further explanations why the long-term follow-up frequency is low particularly in Finland. Most of the patients have only mild symptoms, reflecting the short diagnostic delay and good recognition of the disease by physicians (27). This, along with the good dietary adherence, has made severe complications associated with advanced or poorly treated coeliac disease rare (4). As a result, demonstration of initial treatment response might often be deemed sufficient by physicians. From patients' perspective, they may find it hard to motivate themselves to seek for healthcare visits when the disease does not constitute a substantial daily burden. In Finland public healthcare is accessible equally to all citizens, further indicating that the patients themselves have not contacted the healthcare services. We believe that patients would have received a follow-up if actively seeking it. Thus, it is likely that, even if patients would "like" to have a follow-up, they do not actively seek it if not organized by the health care. In these circumstances, a more active role of the healthcare providers might be required to improve attendance in follow-up (28).

One of the main arguments for follow-up in coeliac disease is the need to monitor dietary adherence. It was thus of significance that also patients without follow-up reported excellent adherence and competence to maintain a gluten-free diet. This was supported by the low frequency of current coeliac antibody positivity, although it must be realized that serology has limited sensitivity in the detection of dietary lapses (3). The higher prevalence of seropositivity over against self-reported adherence could be caused by unrecognized lapses or via the slow normalization of high values. $(13,29-31)$. Previously Bardella et al. found regular follow-up to improve adherence in adults (13) and Barnea et al. reported similar finding in children (29). The absence of such an association in Finland could be due to the high prevalence of the disease and good availability of gluten-free products, and the widespread knowledge of 
coeliac disease among physicians and food industry. We also wish to emphasize that previously all Finnish patients received governmental reimbursement for dietary expenses (ended in 2016). Owing to these particularities, our results may not be directly applicable to countries in which coeliac disease is rarer and less well known.

The majority of patients were satisfied that the follow-up was arranged by general practitioners, although one third would prefer visits to a specialist. This is in line with the strong public healthcare sector in Finland and a long tradition of decentralization in coeliac disease follow-up. This aspect has not been studied using a similar approach, but Bebb et al. (26) found the preferred method for follow-up to be visits to a dietician with a possibility to consult a physician. We find such a dietician-led clinic an interesting alternative, as dietary lapses are the most common reason for non-response in coeliac disease, even in apparently compliant subjects (32). However, this approach would likely provide no major benefits in well-doing and strictly adherent patients. Anyhow, the possible need for patients with persistent symptoms to consult a physician should be taken into account in these circumstances.

Although most of the long-term outcomes were comparable between the study groups, patients without follow-up felt they had more current symptoms when asked as a qualitative estimation. We recently found persistent symptoms to be common even in welltreated coeliac disease (33), but no studies have looked into the association between ongoing symptoms and lack of follow-up. Interestingly, the difference in symptoms disappeared when evaluated by a validated questionnaire, indicating that the direct question emphasizes the subjective experience rather than the objective magnitude of symptoms. Other explanation for the results is that GSRS measures a wide scale of even opposing symptoms such as diarrhea and constipation. Gastrointestinal symptoms are common and not always necessarily related to coeliac disease. In fact, the median GSRS scores in our treated celiac disease patients were comparable to the Finnish population (33). It might be that regular healthcare contacts improve 
sense of self-capability and reduce excessive monitoring of symptoms. Accordingly, patients with irritable bowel syndrome have benefited from physicians' reassurance on the benignity of their symptoms $(34,35)$.

Strength of the present study was the large number of patients diagnosed on different levels of healthcare. We also succeeded in collecting a wide variety of clinically relevant parameters, and the use of validated questionnaires increases the reliability of results. As a limitation, patient recruitment by newspaper advertisements and via celiac societies predisposes to selection bias. For example, the study information might have reached different age groups and socioeconomic classes unequally. Furthermore, although not specifically designed as a follow-up study, patients without regular follow-up could have considered our survey as an "extra visit" and thus have been more eager to participate. Additional study limitations were the lack of data on the patients' socio-economic status $(30,36)$, scarcity of short-term serological follow-up data and the use of inexact three-point scale in the evaluation of self-reported symptoms. The cross-sectional design also prevented us from evaluating the causality in for example the incidence of comorbidities.

In conclusion, we found follow-up in coeliac disease not to meet the current recommendations. However, the necessity for rigid follow-up scheme could be questioned, as there was no difference between the study groups in most long-term health outcomes. These findings call for more a personalized approach: patients achieving clear remission could visit healthcare only if symptoms reappear or after major life changes, whereas those facing more challenges might benefit from more systematic follow-up. 


\section{References}

1. Lohi S, Mustalahti K, Kaukinen K, Laurila K, Collin P, Rissanen H, et al. Increasing prevalence of coeliac disease over time. Aliment Pharmacol Thera 2007;26:1217.

2. Ludvigsson JF, Bai JC, Biagi F, Card TR, Ciacci C, Ciclitira PJ, et al. Diagnosis and management of adult coeliac disease: guidelines from the British Society of Gastroenterology. Gut 2014;63:1210-1228.

3. Vahedi K, Mascart F, Mary JY, Laberenne JE, Bouhnik Y, Morin MC, et al. Reliability of antitransglutaminase antibodies as predictors of gluten-free diet compliance in adult celiac disease. Am J Gastroenterol 2003;98:1079-1087.

4. Ilus T, Kaukinen K, Virta LJ, Huhtala H, Mäki M, Kurppa K, et al. Refractory coeliac disease in a country with a high prevalence of clinically-diagnosed coeliac disease. Aliment Pharmacol Ther 2014;39:418-425.

5. Lebwohl B, Granath F, Ekbom A, Smedby KE, Murray JA, Neugut AI, et al. Mucosal healing and risk for lymphoproliferative malignancy in celiac disease. Ann Intern Med 2013;159:60.

6. Tio M, Cox MR, Eslick GD. Meta-analysis: coeliac disease and the risk of all-cause mortality, any malignancy and lymphoid malignancy. Aliment Pharmacol Ther 2012;35:540551.

7. Kaukinen K, Peräaho M, Lindfors K, Partanen J, Woolley N, Pikkarainen P, et al. Persistent small bowel mucosal villous atrophy without symptoms in coeliac disease. Aliment Pharmacol Ther 2007;25:1237-1245.

8. Bai JC, Fried M, Corazza GR, Schuppan D, Farthing M, Catassi C, et al. World Gastroenterology Organisation global guidelines on celiac disease. J Clin Gastroenterol 2013;47:121-126. 
9. Villafuerte-Galvez J, Vanga RR, Dennis M, Hansen J, Leffler DA, Kelly CP, et al. Factors governing long-term adherence to a gluten-free diet in adult patients with coeliac disease. Aliment Pharmacol Ther 2015;42:753-760.

10. Annibale B, Severi C, Chistolini A, Antonelli G, Lahner E, Marcheggiano A, et al. Efficacy of gluten-free diet alone on recovery from iron deficiency anemia in adult celiac patients. Am J Gastroenterol 2001;96:132-137.

11. Herman ML, Rubio-Tapia A, Lahr BD, Larson JJ, Van Dyke CT, Murray JA. Patients with celiac disease are not followed up adequately. Clin Gastroenterol Hepatol 2012;10:893. 12. Leffler D, Edwards-George J, Dennis M, Schuppan D, Cook F, Franko D, et al. Factors that influence adherence to a gluten-free diet in adults with celiac disease. Dig Dis Sci 2008;53:1573-1581.

13. Bardella MT, Molteni N, Prampolini L, Giunta AM, Baldassarri AR, Morganti D, et al. Need for follow up in coeliac disease. Arch Dis Child 1994;70:211-213.

14. Haines ML, Anderson RP, Gibson PR. Systematic review: The evidence base for longterm management of coeliac disease. Aliment Pharmacol Ther 2008;28:1042-1066.

15. Hall NJ, Rubin G, Charnock A. Systematic review: adherence to a gluten-free diet in adult patients with coeliac disease. Aliment Pharmacol Ther 2009;30:315-330.

16. Pekki H, Kurppa K, Mäki M, Huhtala H, Laurila K, Ilus T, et al. Performing routine follow-up biopsy 1 year after diagnosis does not affect long-term outcomes in coeliac disease. Aliment Pharmacol Ther 2017;45:1459-1468.

17. Kivelä L, Kaukinen K, Lähdeaho ML, Huhtala H, Ashorn M, Ruuska T, et al. Presentation of celiac disease in Finnish children is no longer changing: A 50-Year perspective. J Pediatr 2015;167:1109-1115. 
18. Kurppa K, Paavola A, Collin P, Sievänen H, Laurila K, Huhtala H, et al. Benefits of a gluten-free diet for asymptomatic patients with serologic markers of celiac disease. Gastroenterology 2014;147:610-617.

19. Haere P, Hoie O, Schulz T, Schonhardt I, Raki M, Lundin KE. Long-term mucosal recovery and healing in celiac disease is the rule - not the exception. Scand J Gastroenterol $2016 ; 18: 1-8$

20. Hallert C, Granno C, Hulten S, Midhagen G, Strom M, Svensson H, et al. Living with coeliac disease: controlled study of the burden of illness. Scand J Gastroenterol 2002;37:3942.

21. McHorney CA, Ware JE,Jr, Lu JF, Sherbourne CD. The MOS 36-item Short-Form Health Survey (SF-36): III. Tests of data quality, scaling assumptions, and reliability across diverse patient groups. Med Care 1994;32:40-66.

22. Viljamaa M, Collin P, Huhtala H, Sievänen H, Mäki M, Kaukinen K. Is coeliac disease screening in risk groups justified? A fourteen-year follow-up with special focus on compliance and quality of life. Aliment Pharmacol Ther 2005;22:317-324.

23. Hallert C, Granno C, Grant C, Hulten S, Midhagen G, Strom M, et al. Quality of life of adult coeliac patients treated for 10 years. Scand J Gastroenterol 1998;33:933-938.

24. Dimenas E, Carlsson G, Glise H, Israelsson B, Wiklund I. Relevance of norm values as part of the documentation of quality of life instruments for use in upper gastrointestinal disease. Scand J Gastroenterol 1996;221:8-13.

25. Bardella MT, Velio P, Cesana BM, Prampolini L, Casella G, Di Bella C, et al. Coeliac disease: a histological follow-up study. Histopathology 2007;50:465-471.

26. Bebb JR, Lawson A, Knight T, Long RG. Long-term follow-up of coeliac disease--what do coeliac patients want? Aliment Pharmacol Ther 2006;23:827-831. 
27. Ukkola A, Mäki M, Kurppa K, Collin P, Huhtala H, Kekkonen L, et al. Diet Improves perception of health and well-being in symptomatic, but not asymptomatic, patients with celiac disease. Clin Gastroenterol Hepatol 2011;9:118-123.

28. Artama M, Heinavaara S, Sarkeala T, Prattala R, Pukkala E, Malila N. Determinants of non-participation in a mass screening program for colorectal cancer in Finland. Acta Oncol 2016;55:870-874.

29. Barnea L, Mozer-Glassberg Y, Hojsak I, Hartman C, Shamir R. Pediatric celiac disease patients who are lost to follow-up have a poorly controlled disease. Digestion 2014;90:248253.

30. Leffler DA, Edwards George JB, Dennis M, Cook EF, Schuppan D, Kelly CP. A prospective comparative study of five measures of gluten-free diet adherence in adults with coeliac disease. Aliment Pharmacol Ther 2007;26:1227-1235.

31. Zanini B, Lanzarotto F, Mora A, Bertolazzi S, Turini D, Cesana B, et al. Five year time course of celiac disease serology during gluten free diet: results of a community based "CDWatch" program. Dig Liver Dis 2010;42:865-870.

32. Leffler DA, Dennis M, Hyett B, Kelly E, Schuppan D, Kelly CP. Etiologies and predictors of diagnosis in nonresponsive celiac disease. Clin Gastroenterol Hepatol 2007;5:445-450.

33. Laurikka P, Salmi T, Collin P, Huhtala H, Mäki M, Kaukinen K, et al. Gastrointestinal symptoms in celiac disease patients on a long-term gluten-free diet. Nutrients 2016;8:e429.

34. Spiegel BM, Gralnek IM, Bolus R, Chang L, Dulai GS, Mayer EA, et al. Clinical determinants of health-related quality of life in patients with irritable bowel syndrome. Arch Intern Med 2004;164:1773-1780. 
35. Lackner JM, Gudleski GD, Ma CX, Dewanwala A, Naliboff B, Representing the IBSOS Outcome Study Research Group. Fear of GI symptoms has an important impact on quality of life in patients with moderate-to-severe IBS. Am J Gastroenterol 2014;109:1815-1823.

36. Ciacci C, Cirillo M, Cavallaro R, Mazzacca G. Long-term follow-up of celiac adults on gluten-free diet: prevalence and correlates of intestinal damage. Digestion 2002;66:178-185. 
Figure legends

Figure 1: Patients' opinion on where the long-term follow-up of celiac disease should be organized, divided on basis of the lack (No follow-up) or presence (Follow-up) of current regular follow-up.

Figure 2. Patients' opinion on who should be in charge of the long-term follow-up of celiac disease, divided on basis of the lack (No follow-up) or presence (Follow-up) of current regular follow-up. 